\title{
$¿$ ¿Son adictos a las redes sociales los estudiantes universitarios?
}

\section{Are they adicctied to college students social networks?}

\author{
Verónica MARÍN DÍAZ, Begoña Esther SAMPEDRO REQUENA \\ y Juan Manuel MUÑOZ GONZÁLEZ \\ Universidad de Córdoba
}

Recibido: Septiembre 2014

Aceptado: Enero 2015

\section{Resumen}

El desarrollo de Internet y más concretamente de las redes sociales ha supuesto dar una nueva visión a los diferentes aspectos que giran alrededor de la vida de los sujetos, siendo uno de ellos las adicciones, y más concretamente las que se pueden presentar en torno a las tecnologías. En este estudio presentamos los resultados de una investigación realizada entre alumnado universitario de la macroárea de Ciencias Sociales y Jurídicas sobre la adicción de estos a Internet en general y a las redes sociales en particular, todo ello a través de un diseño descriptivo correlacional. La muestra participante estuvo conformada por 373 sujetos de las provincias de Granada, Sevilla, Málaga y Córdoba. Para ello se ha empleado el cuestionario diseñado por Young, el cual ha sido traducido al español. El objetivo principal de partida ha sido determinar si los estudiantes universitarios pueden ser considerados adictos a las redes sociales. El resultado más destacado es que los alumnos de las titulaciones estudiadas no sienten ser adictos a Internet ni a las redes sociales, en concreto las mujeres reflejan una mayor distancia con las redes. Es significativo que los datos logrados difieren de los encontrados en la literatura revisada. Por lo que cabe preguntarse si el alumnado se encuentra en la fase de negación de la adicción.

Palabas clave: internet, adicción, estudiante universitario, redes sociales.

\begin{abstract}
The development of the Internet and in particular of social networks has supposedly given a new view to the different aspects that surround human behavior. It includes those associated with addictions, but specifically the ones that have to do with technologies. Following a correlational descriptive design we present the results of a study, which involved university students from Social and Legal Sciences as participants, about their addiction to the Internet and in particular to social networks. The sample was conformed of 373 participants from the cities of Granada, Sevilla, Málaga, and Córdoba. To gather the data a questionnaire that was design by Young was translated to Spanish. The main research objective was to determine if university students could be considered social network addicts. The most prominent result was that the participants don't consider themselves to be addicted to the Internet or to social networks; in particular women reflected a major distance from the social networks. It's important to know


that the results differ from those found in the literature review, which opens the question, are the participants in a phase of denial towards the addiction?

Keywords: internet, addiction, university students, social network.

El desarrollo de Internet o como algunos la denominan Web 2.0 (O’Reilly, 2005) es una realidad hoy incuestionable, al igual que lo es su presencia en la vida profesional y personal de los individuos. Como señalan Bennett, Bishop, Dalgarno, Waycott y Kennedy (2012) la emergencia con la que ha crecido la Web 2.0 ha promovido nuevas oportunidades, no solo para crear, sino también para compartir e interactuar con otros sujetos. Este último aspecto se ha materializado en diversas herramientas que han crecido bajo su paraguas, como pueden ser videojuegos, blogs, wikis, repositorios de fotos, de videos o las redes sociales.

En el ámbito social y educativo todas ellas han ido cobrando una gran relevancia, llegando incluso a convertirse en un elemento más de las dinámicas diarias (Akçay y Arslan, 2010; Bullen, Morgan y Qayyum, 2011; Marín, 2014). En lo que se refiere a este último vemos, pues, que sus principales potenciales radican en posibilitar la interacción directa docente-alumno en cualquier momento y desde cualquier lugar, hacer más atractivo el aprendizaje o la creación de comunidades de aprendizaje, en las cuales se comparte un interés común y que permite profundizar en el conocimiento del mismo y potenciar la inteligencia colectiva (Trujillo, 2011).

No obstante, no todo lo que ofrece Internet al mundo social y educativo, en este caso, está libre de generar situaciones problemáticas o de peligro, tales como dispersar la atención, reducir la productividad, suplantar la identidad, potenciar conductas individualistas, adicción a ellas, degradación del lenguaje, nuevos tipos de delitos informáticos, ... (Caldevilla, 2010). Por tanto, hoy uno de los problemas que más presencia está teniendo en torno a este medio en general y a las redes sociales en particular es la adicción. Según Watters, Keefer, Kloosterman, Summerfeldt y Parker (2013) la adicción a Internet se puede entender como el uso compulsivo y excesivo de esta, provocando un malestar significativo así como un deterioro en el funcionamiento diario del individuo. Un ejemplo de ello lo encontramos en el estudio realizado por Lam, Peng, Maü y Jing (2009), quienes señalan cómo al ir aumentando la presencia de Internet, así como de todas sus herramientas, en el devenir diario de los sujetos, ha ido provocando nuevas adicciones, siendo los videojuegos y las redes sociales los que más adictos están generando.

Es en esta última donde centraremos nuestro trabajo, en determinar si la actividad de los estudiantes universitarios en las redes sociales hace o provoca que estos sean adictos a ellas. Pero, ¿qué hace que las redes sociales puedan generar situaciones de adicción entre los jóvenes? ¿Por qué provocan que los jóvenes de hoy deseen pertenecer a una de ellas? ¿Por qué emplean un gran número de horas de su tiempo en ellas? Si atendemos a las características que Campos $(2008,2)$ aportaba como rasgos definitorios de estas, "concepto de comunidad, a través de la creación de redes de usuarios que interactúan, dialogan y aportan comunicación y conocimiento; tecnología flexible y ancho de banda necesario para el intercambio de información y estándares web de aplicación libre; y una arquitectura modular que favorece la creación de 
aplicaciones complejas de forma más rápida, a un menor coste”, podemos considerar que son un servicio online en él cual se produce una comunicación social y donde, a través de las relaciones, se comparte información personal (Tiryakioglu y Erzurum, 2011), Por otra parte ¿Por qué centrarnos en este colectivo?, la razón es muy sencilla, en la actualidad la presencia de los jóvenes, que hoy realizan estudios universitarios, en las redes sociales académicas o no, es cada vez mayor, tal y como refleja el estudio realizado en 2011 por EDUCAUSE, donde se señalaba como el 90\% de los universitarios estadounidenses participaba en alguna red social. $O$ en el caso de España, donde el $73.84 \%$ de los alumnos que cursaban enseñanza superior prefieren las redes sociales para la docencia universitaria (Tuñez y Sixto, 2012), Por otra parte, son significativos los datos recogidos en el estudio realizado por Bernal y Angulo (2013), en el cual se refleja que el inicio de los jóvenes en las redes sociales es más temprano, situando la media de edad en 12 años, aspecto este inquietante y que provoca que nos planteemos hasta dónde llega la dependencia de los jóvenes por este medio.

\section{Método}

A través de un método de investigación de tipo ex post facto, siendo concretamente su diseño de carácter descriptivo y correlacional atendiendo a la clasificación que realiza Mateo (2012, p.196) se ha procedido a establecer los objetivos de partida, siendo este el determinar si los estudiantes universitarios pueden ser considerados adictos a las redes sociales. Las hipótesis propuestas a partir del mencionado objetivo, se agrupan en estadísticas y de investigación, estas últimas formuladas en términos de interrogante, y las primeras en forma de análisis matemático.

Interrogantes:

- ¿ ¿Son adictos los estudiantes a las redes? Y si fuera así, ¿̇on conscientes de ello?,

- ¿El uso de Internet implica el consumo de productos como las redes sociales?

- ¿Pasan demasiado tiempo los estudiantes universitarios navegando en las redes?

- ¿El uso y consumo de las redes sociales afecta a sus estudios y vida social fuera de la red?

Estadísticas:

- Existen diferencias estadísticamente significativas en el consumo de las redes a favor de las mujeres 


\section{Instrumento de recogida de datos.}

Para la recogida de datos se ha empleado la técnica de la encuesta, y dentro de ella se optó por diseñar un cuestionario en formato online, apoyado en el instrumento creado por Kimberly Young referido a la adicción a Internet y disponible en http://netaddiction.com/internet-addiction-test/ (Watters, Keefers et al. 2013).

Tras la autorización de los autores para su traducción y reutilización, se reelaboro el instrumento orientándolo, en este caso, hacia las redes sociales, además de incorporar nuevos ítems, como es el caso de las variables de identificación; además de la edad y el sexo, presentes en la versión original, se incluyó la titulación que estuvieran cursando en el momento de implementar el cuestionario, curso, ciudad universitaria, posesión de perfil en una red social y si la respuesta a este último ítem era positiva debían responder a la pregunta en cuáles. Asimismo, también se rediseñaron los ítems orientados a las redes sociales en lugar de a Internet.

Finalmente el instrumento estuvo formado por 27 ítems, donde 8 correspondían a variables dependientes y 19 a las independientes. La escala de repuesta de las primeras era nominal y en el caso de las segundas de escala tipo Likert, donde 1 correspondía a nunca y 5 siempre.

Para comprobar que una vez traducido el instrumento e incorporado los nuevos ítems este respondía tanto al objetivo general como a los interrogantes de partida, se sometió a pruebas de validez de contenido y de constructo y fiabilidad.

En el caso de la validez de contenido se ha empleado la prueba denominada "juicio de expertos". Para ello se contactó con docentes que cumplieran los siguientes requisitos:

Experiencia en el uso y consumo de las TIC, experiencia docente en el ámbito de las TIC, conocimientos de la lengua inglesa de manera fluida (B2), dado que en este caso se les facilitaba el instrumento original en su versión inglesa con el objeto de cotejar las posibles interpretaciones erróneas que se pudieran haber realizado, impartir docencia en titulaciones del área de las Ciencias Sociales y Jurídicas. Finalmente el grupo de expertos estuvo conformado por 9 docentes, de los cuales 3 eran mujeres y 6 hombres, quedando distribuidas su presencia como puede consultarse en la tabla 1.

\begin{tabular}{ccccc}
\hline \multicolumn{5}{c}{ Universidad } \\
\hline & Córdoba & Granada & Málaga & Sevilla \\
\hline Mujer & 2 & 1 & 0 & 0 \\
\hline Hombre & 2 & 1 & 1 & 2 \\
\hline
\end{tabular}

Tabla 1. Distribución de los expertos

Todos ellos poseían un nivel fluido de inglés, una experiencia docente entre 6 y más de 15 años en el ámbito de las tecnologías, así como en el uso y consumo de las TIC.

La valoración de los jueces fue positiva, por lo que no hubo que hacer ninguna modificación

Para determinar la fiabilidad del instrumento se ha procedido a efectuar la prueba alfa de Cronbach. Realizada esta para todo el cuestionario, se obtuvo una fiabilidad de 
0,878, la cual según Mateo (2012) puede ser considerada de alta. Ejecutada la discriminación ítem por ítem, como se puede consultar en la tabla 2, esta ha oscilado entre 0,868 y 0,889 , por lo que se puede confirmar que el instrumento posee una alta fiabilidad o consistencia.

\begin{tabular}{cc}
\hline \multicolumn{2}{c}{ Alfa de Cronbach } \\
\hline Ítem 9 & 0.878 \\
Ítem 10 & 0,872 \\
Ítem 11 & 0,878 \\
Ítem 12 & 0,871 \\
Ítem 13 & 0,876 \\
Ítem 14 & 0,872 \\
Ítem 15 & 0,868 \\
Ítem 16 & 0,884 \\
Ítem 17 & 0,870 \\
Ítem 18 & 0,871 \\
Ítem 19 & 0,889 \\
Ítem 20 & 0,869 \\
Ítem 21 & 0,871 \\
Ítem 22 & 0,873 \\
Ítem 23 & 0,871 \\
Ítem 24 & 0,869 \\
Ítem 25 & 0,870 \\
Ítem 26 & 0,869 \\
Ítem 27 & 0,868 \\
\hline 2. Discriminación ítem por ítem
\end{tabular}

\section{Población y muestra}

La población de partida estaba constituido por todos los estudiantes de los grados de Educación Infantil, Educación Primaria, Educador Social, Pedagogía y Dirección y Gestión de Empresas de las Universidades de Córdoba, Granada, Málaga, Murcia y Sevilla. La muestra total participante estuvo formada por aquel alumnado que se encontraba en el aula el día que se administró el instrumento, quedando compuesta finalmente por 373 sujetos, donde 75 eran hombres, 298 mujeres. Atendiendo a los datos aportados por Gialamas, Nikiolopoulu y Koutromanos (2013) y Cheng y Chang (2006), se puede afirmar que no existe posibilidad de sesgo en la selección de la muestra, dado que tradicionalmente los estudios del campo de las Ciencias Sociales y Jurídicas se han feminizado.

En función de la edad, la muestra se distribuyó tal y como se puede consultar en la tabla 3, quedando la mayor parte de está situada entre los 20, 22 y 23 años respectivamente 
Verónica Marín Díaz et al. ¿ ¿Son adictos a las redes sociales los estudiantes universitarios?

\begin{tabular}{ccc}
\hline Años & Frecuencia & Porcentaje \\
\hline 19 & 11 & 2,9 \\
20 & 97 & 26,0 \\
21 & 47 & 12,6 \\
22 & 60 & 16,1 \\
23 & 52 & 13,9 \\
24 & 46 & 12,3 \\
25 & 39 & 10,5 \\
\hline
\end{tabular}

Tabla 3. Distribución de la muestra en función de la edad

En lo que se refiere a la distribución en función de la universidad de procedencia, el 37,8\% era de la Universidad de Granada, el 31,9\% de la de Córdoba, el 18,8\% de Málaga, 9,4 y el 0.3 de Sevilla y Murcia respectivamente. Atendiendo al grado cursado, el 53,9\% realiza los estudios de Educación Infantil, el 19\% de Dirección y Gestión de Empresas, 20,4\% en el Grado de Pedagogía, el 4\% de Educación Primaria y el 2,7\% Educador Social. En función del curso que se encontraban cursando en el momento de realizar el cuestionario, la mayoría se encontraba en segundo curso de carrera (43,4\%), seguidos de tercero (22,5\%), cuarto $(19,3 \%)$ y primero $(14,7 \%)$,

Realizada la contingencia sexo-curso académico y curso en proceso-titulación (ver tablas 4 y 5 gráfica 1), se encuentra que la mayoría de la muestra son mujeres que están cursando segundo curso del Grado de Educación Infantil y tercero de Pedagogía.

\begin{tabular}{ccccc}
\hline \multirow{2}{*}{ Género } & \multicolumn{4}{c}{ Curso } \\
\cline { 2 - 5 } & Primero & Segundo & Tercero & Cuarto \\
\hline Mujer & 10 & 31 & 19 & 15 \\
\hline Hombre & 45 & 131 & 65 & 57 \\
\hline
\end{tabular}

Tabla 4. Contingencia sexo-curso

\begin{tabular}{lccccc}
\hline \multirow{2}{*}{ Curso } & \multicolumn{5}{c}{ Titulación o estudios en proceso } \\
\cline { 2 - 6 } & $\begin{array}{c}\text { G, de E. } \\
\text { Infantil }\end{array}$ & $\begin{array}{c}\text { G. de E. } \\
\text { Primaria }\end{array}$ & $\begin{array}{c}\text { G. de E. } \\
\text { Social }\end{array}$ & $\begin{array}{c}\text { G. de } \\
\text { Pedagogía }\end{array}$ & $\begin{array}{c}\text { G. en D. y G. de } \\
\text { Empresas }\end{array}$ \\
\hline Primero & 34 & 7 & 6 & 8 & 0 \\
Segundo & 77 & 3 & 2 & 36 & 44 \\
Tercero & 41 & 2 & 1 & 26 & 14 \\
Cuarto & 49 & 3 & 1 & 6 & 13 \\
\hline
\end{tabular}

Tabla 5. Contingencia Curso-Titulación o estudios en procesos 
Verónica Marín Díaz et al. ¿ ¿Son adictos a las redes sociales los estudiantes universitarios?

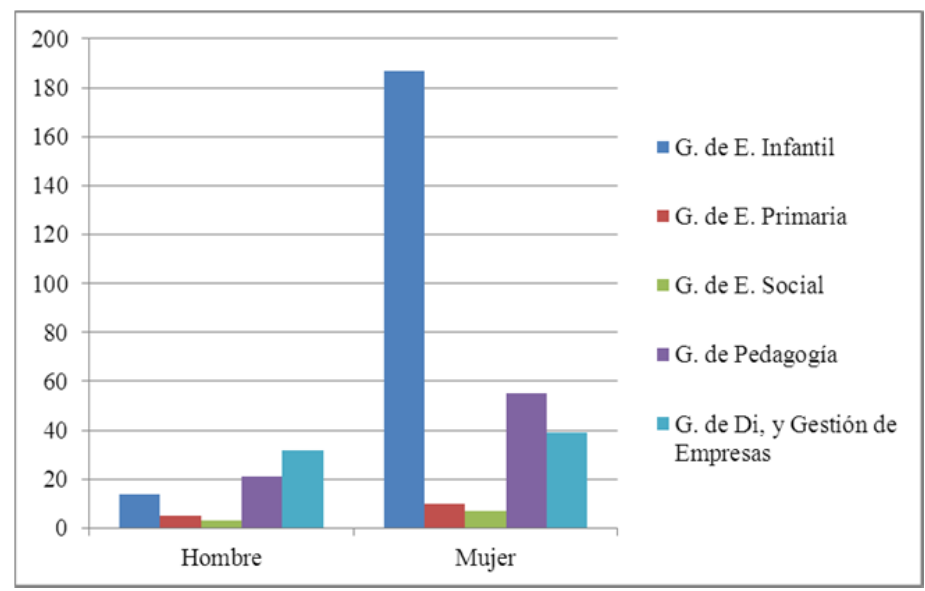

Gráfica 1. Contingencia Sexo-Titulación o estudios en proceso

Con respecto al ítem referido a si poseen un perfil en una red social, el 97,7\% de la muestra contesta de forma positiva, indicando que este se encuentra distribuido entre las siguientes redes sociales: Facebook un 56,6\%, Twitter un 26.5\%, Skype 4,8\%, Linkedin un $1,9 \%$ y solo un $2,4 \%$ señala tener perfil en otras redes sociales, pero no indica en cuáles.

\section{Resultados}

\section{Estudio descriptivo}

A continuación presentamos la valoración de los ítems del cuestionario, como puede consultarse en la tabla 6. Los ítems más valorados se encuentran referidos a la frecuencia con la qué prefieren pasar el tiempo conectados a Internet, la frecuencia con la que sus labores educativas se ven afectadas, presentan una actitud agresiva o desafiante tanto cuando están conectados, como cuando no lo están o cuando se les pide que lo dejen, así como estar mal humorados si no están conectados, bloquean ideas o pensamientos que afectan a su vida diaria y provienen de su vida en la red y la frecuencia de tiempo que pasan conectados en vez de estar con sus amigos y compañeros. 


Ítem 9: ¿Con qué frecuencia crees que estás conectado más tiempo de lo previsto?
Ítem 10: ¿Con qué frecuencia descuidas las tareas del hogar para pasar más tiempo en
Internet?

Ítem 11: ¿Con qué frecuencia prefieres pasar el tiempo conectado a Internet?

Media

$\mathrm{S}$

$3,82 \quad 1,150$

Ítem 12: ¿Con qué frecuencia creas nuevas relaciones con otros usuarios de las redes sociales?

Ítem 13: ¿Con qué frecuencia los demás personas de su vida se quejan del tiempo que

4,12

1,082

pasas conectado a las redes sociales?

Ítem 14: ¿Con qué frecuencia tus calificaciones o trabajos universitarios se ven afectados a causa del tiempo que pasas conectado a las redes sociales?

Ítem 15: ¿ Revisas tu correo electrónico antes que otra actividad académica que tengas que

4,72 ,773

$4,14 \quad 1,095$

$4,23 \quad 1,128$

4,52 988

$3,92 \quad 1,248$ hacer?

Ítem 16: ¿Con qué frecuencia tu rendimiento en el los estudios se ven afectados por el tiempo que pasas en las redes sociales?

Ítem 17: ¿Con qué frecuencia te pones a la defensiva o en actitud reservada cuando alguien le pregunta lo que haces en las redes sociales?

Ítem 18: ¿Con qué frecuencia bloqueas pensamientos/ideas de tu vida diaria que te perturban con pensamientos/ideas de lo que sucede en tu vida en la red social?

Ítem 19: ¿Piensas que la vida sin las redes sociales sería aburrida?

Ítem 20: ¿Con qué frecuencia te enfadas cuando alguien te interrumpe cuando estás conectado a las redes sociales?

Ítem 21: ¿Con qué frecuencia pasas la noche o parte de esta conectado a las redes sociales?

Ítem 22: ¿Con qué frecuencia te sientes desconectado cuando no estás conectado a las redes sociales?

Ítem 23: ¿Con qué frecuencia tienes que desconectar de la red social y piensas "unos minutos más"?

Ítem 24: ¿Con qué frecuencia intentas reducir el tiempo que pasas conectado a las redes sociales y no lo logras?

Ítem 25: ¿Cuándo te preguntan el tiempo que pasas conectado a las redes sociales, ocultas el tiempo real de conexión?

Ítem 26: ¿Con qué frecuencia pasas más tiempo conectado a las redes sociales en vez de

Tabla 6. Estudio de la media y Desviación Típica

En lo que se refiere a los ítems que han superado una media de 4,5 (tabla 7), nos encontramos un conjunto de 8 ítems, en los cuales los estudiantes señalan que raramente prefieren pasar el tiempo conectados a Internet $(86,9 \%)$ frente al $0,5 \%$ que piensa con mucha frecuencia que si prefiere ello (ítem 11: Con qué frecuencia prefieres pasar el tiempo conectado a Internet). En la misma línea, la gran mayoría considera que raramente el tiempo que pasan conectados a las redes sociales pueda afectar a sus calificaciones o trabajos universitarios (ítem 14). A la pregunta ¿con qué frecuencia te 
pones a la defensiva o en actitud reservada, cuando alguien le pregunta lo que haces en las redes sociales? (ítem 17), los estudiantes participantes reflejaron en un 75,1\% que raramente se sentían con una actitud defensiva o reservada, frente al 3,5 \% y el 1,3\% que frecuentemente o con mucha frecuencia se siente así.

En lo que se refiere a la frecuencia con la que los estudiantes encuestados bloquean pensamientos/ideas de su vida diaria, que les perturban con pensamientos/ideas de lo que sucede en su vida en la red social (ítem 18), encontramos que el $82,3 \%$ de los participantes raramente se encuentra en dicha situación frente al 0,8 y 0,3\% que con mucha frecuencia o siempre sí.

Siguiendo esta línea, encontramos que el 78,8\% de la muestra raramente se enfada cuando alguien le interrumpe cuando está conectado a las redes sociales (ítem 20), mientras que el $16,1 \%$ de vez en cuando y el 3,5\% frecuentemente. Por otra parte, el $84,7 \%$ afirma que raramente oculta el tiempo que realmente pasa conectado a las redes sociales cuando le preguntan por ello (ítem 25), mientras que el 9,4\% afirma que lo hace de vez en cuando, el 1,1\% frecuentemente, el 1,6\% y el $0,3 \%$ con mucha frecuencia y siempre, respectivamente.

Cuando a la muestra se le ha preguntado si el tiempo que pasa conectado a las redes sociales afecta a salir con sus amigos y conocidos (ítem 26) el 85,6\% afirma que raramente se produce tal situación, frente al 0,3\% quien indica que siempre sucede. Por último la frecuencia con qué se encuentran deprimidos, enfadados o mal humorados si no se conectan a las redes sociales y que esta sensación desaparece cuando se conectan (ítem 27) vemos que la mayoría de los estudiantes participantes señalan que raramente se encuentran así frente al 10.5\% que si se siente de vez en cuando.

\begin{tabular}{cccccc}
\hline & Raramente & $\begin{array}{c}\text { De vez en } \\
\text { cuando }\end{array}$ & Frecuentemente & $\begin{array}{c}\text { Con mucha } \\
\text { frecuencia }\end{array}$ & Siempre \\
\hline Ítem 11 & 324 & 32 & 9 & 2 & \\
& $(86,9 \%$ & $(8,6 \%)$ & $(2,4 \%)$ & $(0,5 \%)$ & \\
Ítem 14 & 293 & 60 & 6 & 3 & \\
& $(78,6 \%)$ & $(16,1 \%)$ & $(1,6 \%)$ & $(0,8 \%)$ & \\
Ítem 17 & 280 & 61 & 13 & 5 & \\
& $(75,1 \%)$ & $(16,4 \%)$ & $(3,5 \%)$ & $(1,3 \%)$ & 1 \\
Ítem 18 & 307 & 48 & 4 & 3 & $(0,3 \%)$ \\
& $(82,3 \%)$ & $(12,9 \%)$ & $(1,1 \%)$ & $(0,8 \%)$ & \\
Ítem 20 & 294 & 60 & 7 & 2 & 1 \\
& $(78,8 \%)$ & $(16,1 \%)$ & $(1,9)$ & $(0,5 \%)$ & \\
Ítem 25 & 316 & 35 & 4 & 6 & $(0,3 \%)$ \\
& $(84,7 \%)$ & $(9,4 \%)$ & $(1,1)$ & $(1,6 \%)$ & 1 \\
Ítem 26 & 323 & 33 & 5 & 2 & $(0,3 \%)$ \\
& $(85,6)$ & $(8,8 \%)$ & $(1,3 \%)$ & $(0,5 \%)$ & 2 \\
Ítem 27 & 314 & 39 & 6 & 3 & $(0,5 \%)$ \\
\hline
\end{tabular}

Tabla 7. Estudio de la frecuencia y \% más destacado 


\section{Estudio inferencial: T de Student y Análisis de varianza de un factor}

Realizada la prueba $\mathrm{t}$ de Student para muestras independientes (n.s. $=0.05$ ), tomando como variable de clasificación el sexo, encontramos la existencia de diferencias significativas en todos los ítems del cuestionario a favor de las mujeres, siendo estás las que presentan un mayor uso de la Internet, de las redes sociales así como una mayor frecuencia a la hora de presentar una actitud negativa ante preguntas como qué hace en la red o cuando se pide que deje la red para realizar otra actividad, confirmando los datos aportados por el estudio de Villadangos y Labrador (2009).

\begin{tabular}{|c|c|c|c|c|c|}
\hline & Género & Media & Desv. típ. & $\mathbf{F}$ & $\mathbf{p}$ \\
\hline \multirow{2}{*}{ Ítem 9} & Hombre & 3,64 & 1,193 & \multirow{2}{*}{ 638 } & \multirow{2}{*}{,636 } \\
\hline & Mujer & 3,86 & 1,137 & & \\
\hline \multirow{2}{*}{ Ítem 10} & Hombre & 4,00 & 1,230 & \multirow{2}{*}{,749 } & \multirow{2}{*}{,559 } \\
\hline & Mujer & 4,15 & 1,042 & & \\
\hline \multirow{2}{*}{ Ítem 11} & Hombre & 4,53 & 1,070 & \multirow{2}{*}{1,953} & \multirow{2}{*}{,101 } \\
\hline & Mujer & 4,77 & ,673 & & \\
\hline \multirow{2}{*}{ Ítem 12} & Hombre & 3,79 & 1,131 & \multirow{2}{*}{ 818 } & \multirow{2}{*}{,514 } \\
\hline & Mujer & 4,23 & 1,069 & & \\
\hline \multirow{2}{*}{ Ítem 13} & Hombre & 4,08 & 1,353 & \multirow{2}{*}{,265 } & \multirow{2}{*}{ 900 } \\
\hline & Mujer & 4,26 & 1,063 & & \\
\hline \multirow{2}{*}{ Ítem 14} & Hombre & 4,21 & 1,339 & \multirow{2}{*}{,748 } & \multirow{2}{*}{,560 } \\
\hline & Mujer & 4,60 & ,864 & & \\
\hline \multirow{2}{*}{ Ítem 15} & Hombre & 3,75 & 1,386 & \multirow{2}{*}{ 977 } & \multirow{2}{*}{ 420 } \\
\hline & Mujer & 3,96 & 1,210 & & \\
\hline \multirow{2}{*}{ Ítem 16} & Hombre & 4,09 & 1,286 & \multirow{2}{*}{,464 } & \multirow{2}{*}{,762 } \\
\hline & Mujer & 4,47 & ,978 & & \\
\hline \multirow{2}{*}{ Ítem 17} & Hombre & 4,25 & 1,220 & \multirow{2}{*}{ 233 } & \multirow{2}{*}{ 919 } \\
\hline & Mujer & 4,58 & ,911 & & \\
\hline \multirow{2}{*}{ Ítem 18} & Hombre & 4,31 & 1,262 & \multirow{2}{*}{5,688} & \multirow{2}{*}{,000 } \\
\hline & Mujer & 4,68 & ,819 & & \\
\hline Ítem 19 & Hombre & 3,97 & 1,252 & 1596 & 175 \\
\hline Item 19 & Mujer & 3,92 & 1,118 & 1,د90 & (1/5 \\
\hline Ítem 20 & Hombre & 4,28 & 1,269 & 1230 & 298 \\
\hline & Mujer & 4,60 & ,860 & & \\
\hline Ítem 21 & Hombre & 3,93 & 1,388 & 1768 & 135 \\
\hline 1ent 21 & Mujer & 4,23 & 1,083 & 1,700 & נטכו, \\
\hline Ítem 22 & Hombre & 4,07 & 1,308 & 2219 & 004 \\
\hline rem 22 & Mujer & 3,99 & 1,109 & 2,219 & , 004 \\
\hline Ítem 23 & Hombre & 4,07 & 1,277 & 331 & 064 \\
\hline & Mujer & 4,17 & 1,131 & & \\
\hline Ítem 24 & Hombre & 4,13 & 1,277 & 090 & 985 \\
\hline 1'ना1 24 & Mujer & 4,34 & 1,046 & (2) & , \\
\hline
\end{tabular}

Tabla 8. ANOVA de un factor 
Verónica Marín Díaz et al. ¿ ¿Son adictos a las redes sociales los estudiantes universitarios?

\begin{tabular}{lrrrrl}
\hline Ítem 25 & $\begin{array}{r}\text { Hombre } \\
\text { Mujer }\end{array}$ & $\begin{array}{r}4,44 \\
4,69\end{array}$ & $\begin{array}{l}1,165 \\
\text {,865 }\end{array}$ & 2,858 & \multirow{2}{*}{024} \\
\hline \multirow{2}{*}{ Ítem 26 } & Hombre & 4,64 &, 936 & \multirow{2}{*}{1,782} & \multirow{2}{*}{, 132} \\
\hline Mujer & 4,75 &, 756 & & \multirow{2}{*}{, 530} \\
\hline
\end{tabular}

Tabla 8. ANOVA de un factor (cont.)

Tras realizar el análisis de la varianza (n.s.=0.05), tomando como variable el género encontramos que hay diferencias significativas a favor de las mujeres en todos los casos menos en dos, referidos a la frecuencia se sienten desconectados cuando no están conectados (ítem 22 ) donde los hombres presentan una mayor presencia (M: 4.07, S: 1.308, siendo $\mathrm{F}=2.219$ y $\mathrm{p}=.004$ )

\section{Correlación Bivariada}

Con respecto a la prueba $r$ de Pearson para determinar la existencia de correlación entre los ítems de la dimensión segunda, podemos consultar los resultados (ver anexo 1). A la vista de los datos podemos afirmar que existe relación entre casi todos los ítems que componen esta dimensión, dado hay nivel de significatividad bilateral, tanto al n.s=0,01 y al n.s. $=0,05$; aunque la relación entre los mismos fluctúa, atendiendo a cada ítem, entre baja y media como señalan Morales (2008); Pérez Juste, García Llamas, Gil Pascual y Galán González (2009) y Mateo (2012),

También encontramos significación a favor del ítem 20 (Con qué frecuencia te enfadas cuando alguien te interrumpe cuando estás conectado a las redes sociales) con respecto a los ítems 21, 22 y 23 (Con qué frecuencia pasas la noche o parte de esta conectado a las redes sociales; Con qué frecuencia te sientes desconectado cuando no estás conectado y Con qué frecuencia tienes que desconectar de la red social y piensas "unos minutos más").

Por último, podemos señalar una relación significativa entre el ítem 24 (Con qué frecuencia intentas reducir el tiempo que pasas conectado a las redes sociales y no lo logras) con el ítem 25 (Cuándo te preguntan el tiempo que pasas conectado a las redes sociales, ocultas el tiempo real de conexión?) y los ítems 26 y 27 (Con qué frecuencia pasas más tiempo conectado a las redes sociales en vez de salir con tus amigos y conocidos y Con qué frecuencia te encuentras deprimido, enfadado o mal humorado si no estás conectado a las redes sociales y esta sensación desaparece cuando te conectas). 


\section{Análisis factorial exploratorio}

Realizado el análisis factorial con la finalidad de extraer las comunalidades posibles entre los diferentes ítems encontramos que estos se agrupan en torno a cuatro factores (ver tabla 9), donde el cuarto factor explica en 51,5015 de la varianza total explicada, el tercero el $46,179 \%$, el segundo el $40,391 \%$ y finalmente el primero explica el $34,021 \%$.

Como vemos el primer factor está conformado por 8 ítems, que presentan un alfa de Cronbach de .887, los cuales giran en torno a la situación personal del estudiante, tales como presentar una actitud defensiva, pensar que pasa más tiempo conectado de lo que debiera, encontrarse deprimido, enfadado o mal (ítems 9, 17, 27, 20, 25,12,11, 21 y 18). El segundo factor está integrado por 4 factores que agrupan a los ítems relacionados con su vida académica (ítems 14, 16, 24 y 13), el cual ha obtenido una fiabilidad de .840. En el factor tercero encontramos los ítems 19, 22, 23, 26 y 10, referidos a su vida en general, obteniendo este factor un alfa de .869. Por último, el cuarto factor está compuesto por un único ítem, el 15, referido al tiempo que dedica a la revisión de correo frente a otra actividad académica, al cual no es posible extraer la fiabilidad, por lo que puede ser eliminado.

\begin{tabular}{|c|c|c|c|c|}
\hline & \multicolumn{4}{|c|}{ Factores } \\
\hline & 1 & 2 & 3 & 4 \\
\hline $\begin{array}{l}\text { Ítem 9: ¿Con qué frecuencia crees que estás conectado más tiempo de lo } \\
\text { previsto? }\end{array}$ & ,659 & & & \\
\hline $\begin{array}{l}\text { Ítem 17: ¿Con qué frecuencia te pones a la defensiva o en actitud reservada } \\
\text { cuando alguien le pregunta lo que haces en las redes sociales? }\end{array}$ & ,649 & & & \\
\hline $\begin{array}{l}\text { Ítem 27: ¿Con qué frecuencia te encuentras deprimido, enfadado o mal } \\
\text { humorado si no estás conectado a las redes sociales y esta sensación desaparece } \\
\text { cuando te conectas? }\end{array}$ & ,640 & & & \\
\hline $\begin{array}{l}\text { Ítem 20: ¿Con qué frecuencia te enfadas cuando alguien te interrumpe cuando } \\
\text { estás conectado a las redes sociales? }\end{array}$ & ,630 & & & \\
\hline $\begin{array}{l}\text { Ítem 25: ¿Cuándo te preguntan el tiempo que pasas conectado a las redes } \\
\text { sociales, ocultas el tiempo real de conexión? }\end{array}$ & ,610 & & & \\
\hline $\begin{array}{l}\text { Ítem 12: ¿Con qué frecuencia creas nuevas relaciones con otros usuarios de las } \\
\text { redes sociales? }\end{array}$ & ,608 & & & \\
\hline Ítem 11: ¿Con qué frecuencia prefieres pasar el tiempo conectado a Internet? & ,572 & & & \\
\hline $\begin{array}{l}\text { Ítem 21: ¿Con qué frecuencia pasas la noche o parte de esta conectado a las } \\
\text { redes sociales? }\end{array}$ &, 530 & & & \\
\hline $\begin{array}{l}\text { Ítem 18: ¿Con qué frecuencia bloqueas pensamientos/ideas de tu vida diaria } \\
\text { que te perturban con pensamientos/ideas de lo que sucede en tu vida en la red } \\
\text { social? }\end{array}$ & ,491 & & & \\
\hline $\begin{array}{l}\text { Ítem 14: ¿Con qué frecuencia tus calificaciones o trabajos universitarios se ven } \\
\text { afectados a causa del tiempo que pasas conectado a las redes sociales? }\end{array}$ & & ,821 & & \\
\hline $\begin{array}{l}\text { Ítem 16: ¿Con qué frecuencia tu rendimiento en el los estudios se ven afectados } \\
\text { por el tiempo que pasas en las redes sociales? }\end{array}$ & & ,785 & & \\
\hline $\begin{array}{l}\text { Îtem 24: ¿Con qué frecuencia intentas reducir el tiempo que pasas conectado a } \\
\text { las redes sociales y no lo logras? }\end{array}$ & & ,459 & & \\
\hline $\begin{array}{l}\text { Ítem 13: ¿Con qué frecuencia los demás personas de su vida se quejan del } \\
\text { tiempo que pasas conectado a las redes sociales? }\end{array}$ & & ,382 & & \\
\hline Ítem 19: ¿Piensas que la vida sin las redes sociales sería aburrida? & & & 684 & \\
\hline
\end{tabular}

Tabla 9. Matriz de componentes rotados 
Ítem 22: ¿Con qué frecuencia te sientes desconectado cuando no estás conectado a las redes sociales?

Ítem 23: ¿Con qué frecuencia tienes que desconectar de la red social y piensas "unos minutos más"?

Ítem 26: ¿Con qué frecuencia pasas más tiempo conectado a las redes sociales en vez de salir con tus amigos y conocidos?

Ítem 10: ¿Con qué frecuencia descuidas las tareas del hogar para pasar más tiempo en Internet?

Ítem 15: ¿Revisas tu correo electrónico antes que otra actividad académica que tengas que hacer?

,603

, 558

, 512

,400

,818

Tabla 9. Matriz de componentes rotados (cont.)

\section{Discusión y conclusiones}

La realidad hoy de nuestros centros de enseñanza superior y de los estudiantes que les dan vida, es que su vida se encuentra vinculada a Internet y más concretamente a las redes sociales (Othman, Suhaimi, Yusuf, Yusuf y Mohamad, 2012), Al igual que el estudio de EDUCAUSE (2011), Baltací-Goktalay y Ozditek (2010), Linne (2015) y de Gómez, Roses y Farrias (2011), los estudiantes universitarios españoles participan de forma activa en Internet y en las redes sociales, dada su alta presencia en las redes más significativas como son Facebook o Twitter, aunque si manifiestan tener cuentas en redes sociales dedicadas fundamentalmente a la búsqueda de trabajo, como es el caso de Linkedin, vemos que a diferencia de los datos aportados por Othman, Suhaimi, et al.(2012), su utilización es muy escasa por parte de los universitarios participantes en el estudio.

Como hemos podido comprobar ¿ $\dot{S}$ Son adictos los estudiantes a las redes? Y si fuera así, ¿'son conscientes de ello? A raíz de los resultados obtenidos podemos afirmar que los estudiantes universitarios españoles no son adictos, a su juicio, de Internet y más concretamente de las redes sociales, como refleja el estudio de Linne (2015). Por otra parte, podemos afirmar, al igual que Gómez, Roses y Farrias (2011), que el uso de estas, sí se encuentran vinculada a aquella. El interrogante ¿pasan demasiado tiempo los estudiantes universitarios navegando en las redes?, confirma que estos consideran no pasar excesivo tiempo y que este no resta tiempo a sus estudios o vida académica tal y como se recoge en el estudio de Eyyam, Menevis y Doçiuer (2010), Tuñez y Sixto (2012) y Caldevilla (2010), ni a su vida personal o social fuera de Internet, de acuerdo con la idea de Muñoz (2010) de que las redes sociales están inmersas de tal modo en nuestras culturas que provocan una inteligencia colectiva, en tanto que podremos afirmar que proveen de oportunidades para el aprendizaje colaborativo y la construcción colectiva del conocimiento (Cress y Kinnenrle, 2008; Laru, Naykki y Jäivelä, 2012) Tampoco encontramos argumentos para señalar que estos estudiantes pueden ser considerados adictos a las redes, dado que no presentan los rasgos adictivos que señalan Durán y Guerra (2015), Echeburría y De Corral (2010), Jiménez-Albiar, Piqueras, Mateu-Martínez, Carballo, Orgiles y Espada (2012). Rial, Gómez, Braña y Varela (2014) y Suría (2012), tales como malestar por no poder conectarse o 
sentimiento de culpa, perder el controlenfadarse por ser interrumpido durante el tiempo que se encuentran conectados, tener trastornos de sueño, presentar un bajo rendimiento en sus estudios o desinterés por sus relaciones sociales fuera de la red y si para afirmar que fomenta la creación de una red de aprendizaje que proporcione autonomía a los miembros (Aguado, 2012). En conclusión, podríamos aventurarnos

Por último, en lo que se refiere a la hipótesis referida a la existencia de diferencias entre hombres y mujeres, en el consumo de Internet y de las redes a favor de las mujeres frente a los hombres al contrario de los resultados alcanzados por Tsai, Liang, Hou y Tsai (2012), en el que reflejaban que los hombres tenían una mejor actitud y presencia ante y en Internet. Los estudios (Booth, Goodman y Kirkup, 2010; JiménezAlbiar, Piqueras, et al, 2012) señalan como las mujeres pasan más tiempo conectadas a las redes, indicando que este lo emplean en establecer relaciones personales, mientras que los hombres prefieren o bien emplear el móvil o bien jugar a videojuegos.

A raíz de todos estos datos cabe preguntarse, si realmente estamos en el primer paso de lo que Echeburúa y De Corral (2010) consideran una adicción a las redes sociales, y que no es más que la negación del hecho de que realmente la vida de los estudiantes universitarios gira en torno a la vida que van construyendo en torno a las redes, pues como aportan Chang y Lee (2013) la confianza que se genera en los amigos virtuales que conforman nuestra red de contactos que reduce tensiones y conflictos y genera estados de cohesión, armonía ,colaboración y cooperación.

\section{Agradecimientos}

Agradecemos a Kateryna Keefer profesora de la Universidad de Trent (Canadá) su autorización para el empleo del instrumento en España.

\section{Referencias bibliográficas}

AGUADO, T. (2012). Redes de cooperación. Espacios de diversidad e igualdad en investigación educativa, en C. Jiménez, L. García, B. Álvarez y J. Quintanal (eds.), Investigación y educación en un mundo en red, 7-17. Madrid: McGraw-Hill

AKÇAY, A. Y ARSLAN, A. (2010). The using of blogs in Turkish education. Procedia Social and Behavioral Sciences, 2, 1195-1199.

BALTACI-GOKTALAY, S. Y OZDITEK, Z. (2010). Preservice teachers' perceptions about Web 2.0 technologies. Procedia Social and Behavioral Sciencies, 2, 47374741.

BENNETT, S., BISHOP, A., DALGARNO, B., WAYCOTT, J. Y KENNEDY, G. (2012). Implementing Web 2.0 technologies in higher education: a collective case study. Computes y Educations, 59, 524-534. Doi: http://dx.doi.org/10.1016/j.compedu.2011.12.022

BERNAL, C. Y ANGULO, J.F. (2013). Interacciones de los jóvenes andaluces en las redes sociales. Comunicar, 40, 25-30. 
Verónica Marín Díaz et al. ¿Son adictos a las redes sociales los estudiantes universitarios?

BOOTH, S., GOODMAN, S. \& KIRKUP, G. (2010). Gender Differences in Learning and working with Technology Social Constructs and cultural context. Hersey: IGI Global.

BULLEN, M., MORGAN, T. Y QAYYUM, A. (2011). Digital learners in higher education: generation is not the issue. Canadian Journal of Learning and Technology, 37 (1), 1-24.

CALDEVILLA, D. (2010). Las redes sociales. Tipologías, uso y consumo de las redes 2.0 en la sociedad digital actual. Documentos de las Ciencias de la Información, 33, 45-61.

CAMPOS, F. (2008). Las redes sociales trastocan los modelos de los medios de comunicación tradicionales. Revista Latina de Comunicación Social, 63. [formato html]

http://www.ull.es/publicaciones/latina/_2008/23_34_Santiago/Francisco_Campos.ht ml (Consultado 12 de agosto de 2010).

CHANG, W.L. Y LEE, C.-Y. (2013). Trust as a learning facilitator that affects students' learning performance in the Facebook community: An investigation in a business planning writing course. Computers y Education, 62, 320-327.

CHENG, J. Y CHANG, C. (2006). Using computers in early childhood classrooms: teachers' attiudes, skills and practices. Journal of Early Childhood Research, 4 (2), 269-288.

CRESS, U. Y KINNENRLE, J. (2008). A systemic and cognitive view on collaborative knowledge bulding with Wikis. International Journal of Computer Supported Collaborative Learning, 3 (2), 105-122.

DURÁN, M Y GUERRA, J.M. (2015). Uso y tendencias adictivas de una muestra de estudiantes universitrios españoles en la red social Tuenti: la actitud positive hacia la presencia de la madre en la red como factor protector. Anales de Psicología, 31 (1), 260-267.

ECHEBURÚA, E. Y DE CORRAL, P. (2010). Adicción a las nuevas tecnologías y a las redes sociales en jóvenes: un nuevo reto. Adicciones, 22 (2), 91-96. [formato html] http://www.adicciones.es/files/91-96\%20editorial\%20echeburua.pdf (Consultado 24 de mayo de 2013).

EDUCAUSE (2011). Ecar national study of undergraduate students and information technology, 2011 report. [formato htm http://www.educause.edu/library/resources/ecar-national-study-undergraduatestudents-and-information-technology-2011-report_(Consultado 4 de junio de 2013).

EYYAM, R., MENEVIS, I. Y DOÇRUER, N. (2010). Perceptions of teacher conductuales towards Web 2.0 technologies. Procedia Social and Behavioral Sciences,15, 2663-2666.

GIALAMAS, V., NIKOLOPOUIOU, K. Y KUTROMANOS, G. (2013). Student teachers' perceptions about the impact of Internet usage on their learning and jobs. 
Verónica Marín Díaz et al. ¿Son adictos a las redes sociales los estudiantes universitarios?

$\begin{array}{llll}\text { Computers } \quad \text { and } \quad \text { Education, } & \text { 1-7. }\end{array}$ http://dx.doi.org/10.1016/j.compedu.2012.10.012

GÓMEZ, M.; ROSES, S. Y FARRIAS, P. (2011). El uso académico de las redes sociales. Comunicar, 38, 131-138. Doi.

JIMÉNEZ-ALBIAR, M $^{\mathrm{a}}$ I., PIQUERAS, J.A., MATEU-MARTÍNEZ, O, CARBALLO, J.L., ORGILES, M. Y ESPADA, J. P. (2012). Diferencia de sexo, caractrística de personalidad y afianzamiento en el uso de Internet, el móvil y los videojuegos en la adolescencia. Health and Addiction, 12 (1), 61-82.

LAM, J.T., PENG, Z-W., MAÜ, J-C. Y JING, J. (2009). Factors associated with Internet addiction among adolescents. CyberPsychology of Behaviour, 12 (5), 551555. Doi: 10.1089/cpb.2009.0036.

LARU, J., NÄYKKI, P. Y JÄVELÄ, S. (2012). Supporting small-group learning using multiple web 2.0 tools: a case study in the higher education context. Internet and Higher Education, 15, 29-38 doi. 10.1016.jhedu.2011.08.004.

LINNE, J. (2015). Estudiar en Internet 2.0 Prácticas de jóvenes universitarios de la Ciudad de Buenos Aires. Comunicación y Sociedad, 23, 195-213

MARÍN, V. (2014). Online Instruction in Spanish Higher Education .A Study Conducted at the Faculty of Education of the University of Córdoba (Spain). The New Educational, 35 (1), 77-87

MATEO, J. (2012). La investigación ex post-facto, en R. Bisquerra (coord.), Metodología de investigación educativa, 195-229. Madrid: La Muralla.

MORALES, P. (2008). Estadística aplicada a las Ciencias Sociales: Correlación y Covarianza. Madrid: Universidad Pontificia Comillas. [formato html] http://web.upcomillas.es/personal/peter/estadisticabasica/correlacion.pdf (Consultado 4 de junio de 2013).

MUÑOZ, G. (2010). Las redes sociales: ¿formula mediática contra la sociedad y el aburrimiento? Revista Latinoamericana de Ciencias Sociales. Niñez y Juventud, 8 (1), 51-64. [formato html] https://www.google.es/url?sa=tyrct=jyq=yesrc=sysource=webycd=1ycad=rjayved= 0CDIQFjAAyurl=http\%3A\%2F\%2Fdialnet.unirioja.es\%2Fdescarga\%2Farticulo\%2 F3254291.pdfyei=OSS_UqzaCcPA0QWJ84DgDgyusg=AFQjCNESPYIoOXqzPX MFl1Px7ieGCC0EuQysig2=ommN9cAgHBVDBCZK509xQybvm=bv.58187178,d.bGQ_(Consultado 15 de agosto de 2014).

O'REILLY, T. (2005). What Is Web 2.0. Design Patterns and Business Models for the Next Generation of Software. http://oreilly.com/web2/archive/what-is-web-20.html (Consultado 15 de agosto de 2014).

OTHAMAN, M. S., SUHAIMI, S., YUSUF, L. M., YUSUF, N. Y MOHAMAD, N. (2012). Analysis of social network categories : social learning and social frienship. Procedia Social and Behavioral Sciences, 56, 441-447. 
Verónica Marín Díaz et al. ¿ ¿Son adictos a las redes sociales los estudiantes universitarios?

PÉREZ JUSTE, R., GARCÍA LLAMAS, J.L.,, GIL PASCUAL, J. A. Y GALÁN GONZÁLEZ, A. (2009). Estadística aplicada a la educación. Madrid: Pearson Educación y UNED.

RIAL, A., GÓMEZ, P., BRAÑA, T. Y VARELA, J. (2014). Actitudes y percepciones y uso de Internet y las redes sociales entre los adolescentes de la comunidad gallega (España). Anales de Psicología, 30 (2), 642-655.

SURIÁ, R. (2012). Discapacidad y adolescencia, ¿̇son factores que potencian el riesgo hacia la adicción a las redes sociales online? XIII Congreso Virtual Interpsiquis. [formato html] http://www.psiquiatria.com/bibliopsiquis/handle/10401/5442 (Consultado 11 de septiembre de 2014).

TSAI, M.-J., LIANG, J.-CH., HOU, H.-T. Y TSAI, CH.-CH. (2012). University students' online information searching strategies in different search contexts. Australasian Journal of Educational Technology, 28 (5), 881-895. http://www.ascilite.org.au/ajet/ajet28/tsai-mj.pdf

TIRYAKIOGIU, F. Y ERZURUM, F. (2011). Use of social networks as an education tool. Contemporary Educational Technology, 2 (2), 135-150.

TRUJILLO, J.M. (2011). Comunicación, innovación, educación y gestión del conocimiento en torno al uso del podcast en la educación superior. RUSC, 8 (2), 6172.

TUÑEZ, M. Y SIXTO, J. (2012). Las redes sociales como entorno docente: análisis de Facobook en la docencia universitaria. Pixel Bit, Revista de Medios y Educación, 41, 77-92.

VILLADANGOS, S. Y LABRADOR, F. (2009). Menores y nuevas tecnologías (NT) ¿uso o abuso?. Anuario de Psicología Clinica de la Salud, 5, 75-83.

WATTERS, C. A., KEEFER, K. V., KLOOSTERMAN, P. H., SUMMERFELDT, L. J. Y PARKER J. D.A (2013). Examining the structure of the Internet Addiction Test in adolescents: A bifactor approach. Computers in Human Behaviour, 29 (6), 2294-2302. DOI: 10.1016/j.chb.2013.05.020. 
Verónica Marín Díaz et al. ¿ ¿Son adictos a las redes sociales los estudiantes universitarios?

\section{Correspondencia con los autores}

Verónica MARÍN DÍAZ

Facultad de Ciencias de la Educación

Avda. San Alberto Magno s/n

14071 Córdoba

e-mail: vmarin@uco.es

Begoña Esther SAMPEDRO REQUENA

Facultad de Ciencias de la Educación

Avda. San Alberto Magno s/n

14071 Córdoba

e-mail: bsampedro@uco.es

Juan Manuel MUÑOZ GONZÁLEZ

Facultad de Ciencias de la Educación

Avda. San Alberto Magno s/n

14071-Córdoba

e-mail: juan.manuel@uco.es 
Verónica Marín Díaz et al. ¿ ¿Son adictos a las redes sociales los estudiantes universitarios?

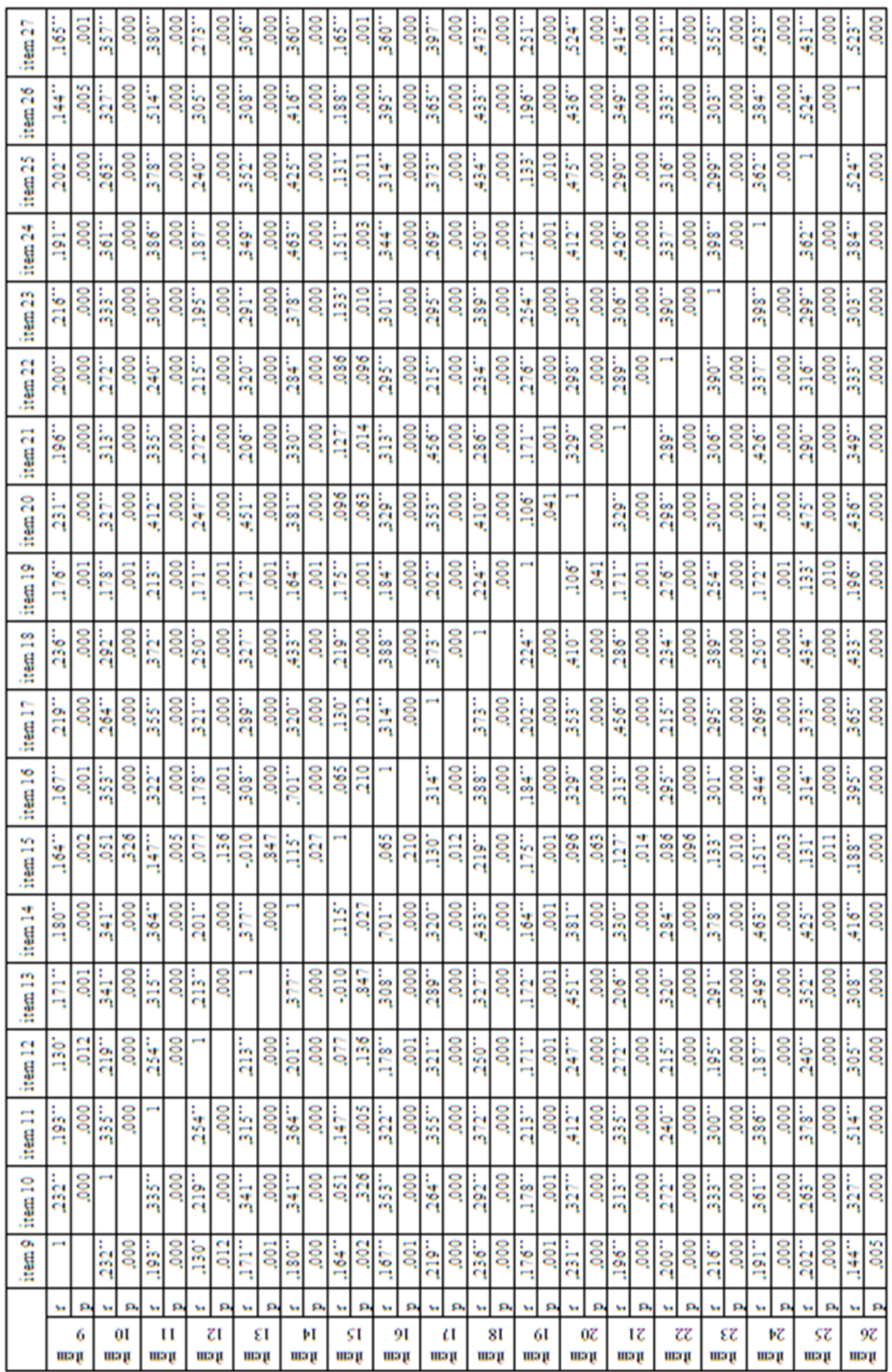

ANEXO 1. Correlaciones bivariadas. 